\title{
Speculative Parallel Execution for Local Timestepping
}

\author{
Maximilian Bremer \\ mb2010@lbl.gov \\ Lawrence Berkeley National Laboratory \\ Berkeley, California, USA \\ Cy Chan \\ cychan@lbl.gov \\ Lawrence Berkeley National Laboratory \\ Berkeley, California, USA
}

\author{
John Bachan \\ jdbachan@lbl.gov \\ Lawrence Berkeley National Laboratory \\ Berkeley, California, USA \\ Clint Dawson \\ clint.dawson@austin.utexas.edu \\ The University of Texas at Austin \\ Austin, Texas, USA
}

\begin{abstract}
Currently, synchronous timestepping for fluid and plasma simulations requires selection of a global time step that conservatively satisfies stability conditions everywhere. However, this approach causes substantial unnecessary work in the presence of large variations of element sizes or local wavespeeds. Local timestepping can significantly reduce work by allowing subdomains to take steps according to local rather than global stability constraints. However, parallelizing this algorithm presents considerable difficulty. Since the stability condition depends on the state of the submesh and its neighbors, dependencies become irregular and may dynamically change as neighbors take smaller or larger timesteps. Furthermore, coarsening and refining timesteps introduces dynamic load imbalance. In order to correctly resolve these dependencies in a distributed setting, we parallelize the local timestepping algorithm using an optimistic (Timewarp-based) parallel discrete event simulation. We introduce waiting heuristics to eliminate misspeculation when dependencies can be identified early, and present a semi-static load balancing strategy to improve scalability. We present detailed performance characterizations of event overheads, misspeculation, and scalability of our approach. Our numerical experiments demonstrate up to a $2.8 \mathrm{x}$ speedup versus a baseline unoptimized approach; a $4 \mathrm{x}$ improvement in per-node throughput compared to an MPI parallelization of synchronous timestepping; and scalability up to 3,072 cores on NERSC Cori's Haswell partition.
\end{abstract}

\section{CCS CONCEPTS}

- Mathematics of computing $\rightarrow$ Partial differential equations; - Computing methodologies $\rightarrow$ Discrete-event simulation; Massively parallel and high-performance simulations; • Applied computing $\rightarrow$ Earth and atmospheric sciences.

\section{KEYWORDS}

Local timestepping; parallel discrete event simulation; Timewarp; shallow water equations; hurricane storm surge

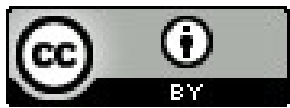

This work is licensed under a Creative Commons Attribution International 4.0 License. SIGSIM-PADS '21, May 31-fune 2, 2021, Virtual Event, USA.

(C) 2021 Copyright held by the owner/author(s).

ACM ISBN 978-1-4503-8296-0/21/05.

https://doi.org/10.1145/3437959.3459257

\section{ACM Reference Format:}

Maximilian Bremer, John Bachan, Cy Chan, and Clint Dawson. 2021. Speculative Parallel Execution for Local Timestepping. In Proceedings of the 2021 ACM SIGSIM Conference on Principles of Advanced Discrete Simulation (SIGSIM-PADS '21), May 31-fune 2, 2021, Virtual Event, USA. ACM, New York, NY, USA, 12 pages. https://doi.org/10.1145/3437959.3459257

\section{INTRODUCTION}

Simulation of systems of hyperbolic conservation laws remains a core area of interest to the scientific computing community, providing insights to fields ranging from computational fluid dynamics to plasma physics. These methods describe the local exchange of conserved quantities such as mass, momentum, and/or energy between regions of space. The celebrated CFL condition has formed the basis of numerous popular explicit timestepping schemes including finite volume methods and discontinuous Galerkin finite element methods. The condition is commonly represented as

$$
\Delta t \leq C_{C F L} \frac{\Delta x}{|\Lambda|},
$$

where $\Delta t$ is the proposed timestep, $\Delta x$ is the length scale, and $|\Lambda|$ is the local wave speed, and $C_{C F L}$ is a discretization-dependent constant. By bounding the allowable timestep as a function of element size and local wave speed, one can limit jumps between neighboring element averages, preventing spurious oscillation.

Traditional synchronous timestepping schemes must guarantee the satisfaction of the CFL condition for all elements. In the presence of large variations of both $\Delta x$ and $|\Lambda|$, high percentages of elements will update with smaller than necessary timesteps. By allowing local regions of the mesh to take different timesteps, the computational work required by the simulation can be significantly reduced.

This paper is part of a larger effort to make the discontinuous Galerkin finite element method a viable alternative to current storm surge simulation codes such as ADCIRC $[7,13,14]$. The discontinuous Galerkin finite element method has advantageous numerical characteristics that improve the fidelity and stability of generated solutions, but currently requires too much computational work to be usable in an operational setting. In a forecasting scenario, the simulation must be able to produce multiple surge forecasts quickly after the hurricane wind forecasts are released to be useful for emergency managers. As a stencil-like kernel, the discontinuous Galerkin finite element method can be parallelized to satisfy the time to solution constraint, but current implementations require too many cores to be practical when running on shared resources 
such as university clusters. Work reduction through local timestepping reduces computational resource requirements, making discontinuous Galerkin storm surge codes competitive for real time forecasting.

Parallelizing adaptive local timestepping (ALTS) is particularly difficult when the propagation speed $\Lambda$ depends on the solution itself. Proposed timestep sizes may change as updates from neighbors are processed. A reduction of an element's timestep may cause a reduction in the neighbor's timestep and a reduction of the neighbor's neighbor's timestep and so on, leading to uncertainty in the number of messages from neighbors that must be processed before a submesh can update. This behavior can be observed, for example, as a tsunami moves inland. In a distributed computing environment, we need to incorporate possibly non-local information when determining if a region can be updated.

The aim of this paper is to optimize the parallel performance of ALTS over core counts relevant for operational surge forecasting. All examples focus on the shallow water equations, the governing equations for storm surge simulation. However, the timestepping scheme should extend directly to arbitrary conservation laws. Dawson et al. examined local parallel timestepping for storm surge in [14]. However, without the capability to dynamically adjust timesteps, the implementation required frequent restarts due to instabilities caused by changing wavespeeds, undermining the stability argument for using discontinuous Galerkin finite elements and limiting performance gains. Following the discrete event-based formulation of [4], we implement ALTS as a discrete event simulation and parallelize it using an optimistic parallel discrete event simulator based on JeffersonâĂŹs Timewarp algorithm. The contributions of this paper are: (1) a set of optimizations to identify and reduce misspeculation; (2) a semi-static load balancer to resolve dynamic load imbalance; and (3) a detailed characterization of the performance of ALTS, including descriptions of event overheads, misspeculation, strong and weak scalability, optimization impact, and comparison versus a state-of-the-art synchronous approach. The parallel efficiency improvements achieved in this work are well suited to enable the use of the discontinuous Galerkin finite element method for operational hurricane storm surge forecasting and other large scale flow simulations which are otherwise intractable due to required computational work.

\subsection{Previous Work}

The idea of allowing different subsystems to advance with different timesteps has been extensively examined for Runge-Kutta methods [17, 29]. Extending these methods to conservation laws has seen considerable work [11, 12, 18, 22, 28, 30]. Additionally, other fields have arrived at related time integration schemes. Quantized state systems (QSS) $[9,15,21]$ have been used to model systems of ordinary differential equations (in particular, for power electronics modeling) by approximating state values with time-dependent polynomials and updating estimates when they deviate more than a given threshold. Those systems focus on enforcing bounds on state variable deviations, as opposed to enforcing particular mathematical constraints, such as a CFL condition. Asynchronous variational integration (AVI) is another locally timestepped method designed for linear elastodynamics [24]. Whereas local timestepping for conservation law is focused on maintaining nonlinear stability, asynchronous variational integration focuses on preserving the total energy of the system.

Beyond the various applications, parallelizations share common motifs based on the characterization of the algorithm. By assuming constant timesteps or variations in timestepping sizes, local timestepping methods can be parallelized using task-graph-like approaches, e.g. $[6,19,31]$. However, the inability to account for variations in the wave speed limits the practicality of these approaches for nonlinear problems. Methods such as quantized state systems and the solver in [25-27] rely on a discrete event simulation framework to account for changes in timestep and correctly resolve dependencies in a parallel context. Our ALTS implementation differs from these other discrete event simulation-based approaches in that: (1) ALTS strictly satisfies the total variation diminishing CFL condition leading to a different control flow and (2) previous approaches have not utilized and examined the performance of ALTS using optimistic parallel discrete event simulation.

\section{OVERVIEW OF ADAPTIVE LOCAL TIMESTEPPING}

In this section, we present a brief overview of the adaptive local timestepping method (ALTS) for conservation laws. The algorithm is designed to ensure that the solution remains free of spurious oscillations by producing a total variation diminishing (TVD) solution [23]. Detailed information on this as well as a proof of correctness of the algorithm are out of scope for this paper, however can be found in [4]. In this section, we provide a detailed description of the control flow, outline a waiting strategy to mitigate bad speculation, describe a semi-static load balancing scheme, and conclude with a discussion of the use of optimistic versus conservative parallel discrete event simulation (PDES) for implementing ALTS.

The simulation of conservation laws using the discontinuous Galerkin finite element or finite volume method models the evolution of densities over a grid. For shallow water flows, we track the evolution of the water column height $h_{j}^{m}$ and the momentum $q_{j}^{m}$, which is the water column height times the depth averaged horizontal velocity. The local wave speed $|\Lambda|$ is derived to be $\sqrt{g h}+|q / h|$, where $g$ is the acceleration due to gravity. We use the $j$ subscript to identify the corresponding element, and $m$ to describe the current timestep. Additionally let $t_{j}^{m}$ denote the simulation time of the $m$ th-update on element $j$. Between successive updates, water and momentum are exchanged between neighboring elements based on a numerical flux. State variables are updated according to net inflow and duration between updates, leading to the conservation of these state variables.

To ensure that the solution is free of oscillations, we require two conditions be satisfied. These restrictions arise due to mathematical constraints, not limitations of the implementation. Firstly, updates must obey the CFL condition, which limits the allowable time between successive updates. Consider an element $j$ and its neighbors $N$, define $t^{*}$ as the most recent time since every element has updated, i.e.

$$
t^{*}=\min _{n \in N} \max _{m}\left\{t_{n}^{m}\right\} .
$$




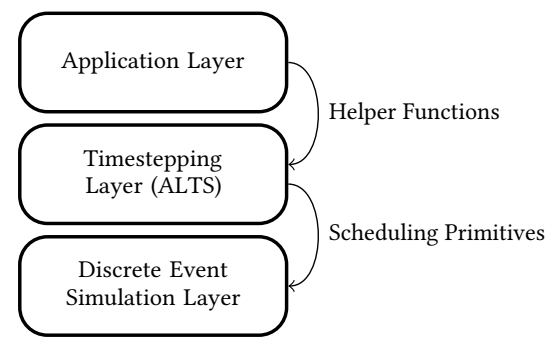

Figure 1: Stack Diagram for Adaptive Local Timestepping

A proposed update advancing element $j$ from $t_{j}^{m}$ to $t_{j}^{m+1}$ satisfies the CFL condition if

$$
t_{j}^{m+1}-t^{*} \leq C_{C F L} \frac{\Delta x_{j}}{\max _{n \in N}\left|\Lambda_{n}\right|(\tau)} \quad \text { for all } t^{*} \leq \tau<t_{j}^{m+1},
$$

where $C_{C F L}$ is a discretization specific constant, $|\Lambda|$ is the largest wavespeed over the stencil, and $\Delta x_{j}$ is the size of the element. This inequality limits the allowable changes in element densities by restricting the timestep size relative to flow rates. Secondly, elements must update in a locally ordered manner. In the ALTS setting, not every element updates at every timestamp. Given two adjacent elements, only one element may update more frequently than the other. To flip which element substeps relative to the other element, the two elements must pass through a synchronization time where both elements update at the same timestamp. We refer to element update traces which obey this behavior as locally ordered.

Before diving into a more detailed description of ALTS, we situate it within the software stack, as shown in Figure 1. As a whole, the ALTS stack is meant as a means for integrating the ordinary differential equation arising after the spatial discretization of arbitrary conservation laws. All physics-related terms are specified at the top-most application layer, such as how to advance the internal state of the submesh between two time points, how to compute local wavespeeds, and how to process halo regions between submeshes. By isolating the physics-specific functionality in the top layer, we allow users to leverage the ALTS framework for multiple applications and reuse existing kernels provided they conform to the helper function API. The application layer combined with the ALTS layer comprise the mathematical model. The ALTS layer expresses the model as a series of time stamped events which are communicated to a general purpose PDES layer via scheduling primitives. The PDES layer serves as the runtime managing the assignment of events to threads and ensuring events are executed in the correct order.

For performance purposes, we agglomerate elements into submeshes that each update as a unit. Submesh updates are limited by the most stringent CFL condition of any element assigned to the submesh, and since elements within the submesh step synchronously, local ordering is trivially satisfied within the submesh. By changing the number of elements per submesh, we gain the ability to tune the computational cost of submesh updates to amortize simulator overheads. As we show later, balancing simulator overheads with computational work is essential to obtaining good performance.

\section{Listing 1: Update event}

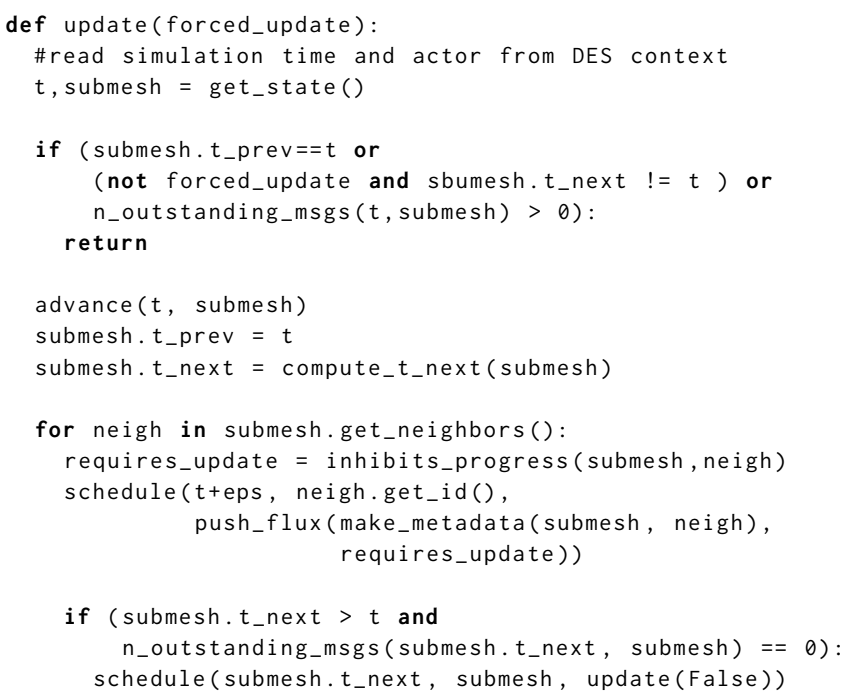

\section{Listing 2: Push flux event}

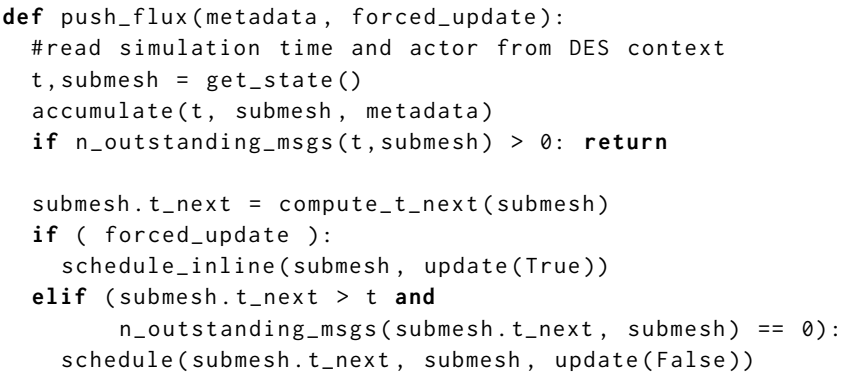

As a discrete event simulation, each actor corresponds to a submesh along with information about neighboring submeshes required to update element densities and compute the CFL condition at the boundary of the submesh. Events are scheduled using a schedule routine which accepts a timestamp, actor, and functor as arguments or can be executed within the same event using a schedule_inline routine. The algorithm consists of two types of events. update advances the submesh state to the current time, and push_flux sends halo data to a neighboring actor. Detailed description of the events is shown in Listing 1 and Listing 2. The eps delay in scheduling the event push_flux is a small amount of subtime used to correctly order updates and push fluxes. The advance, accumulate, and make_meta_data functions encapsulate the mathematical discretization of the conservation law. The halo regions computed as part of make_meta_data for discontinuous Galerkinbased approaches consist of the state variables evaluated at shared interfaces. Higher order finite volume methods would require sending larger halo regions. As presented here, ALTS does assume that the halo region is entirely contained in a single neighboring submesh. The next timestep is computed in compute_t_next to be the largest timestep that satisfies (1). Since the timestep depends on the boundary metadata, the next update time may change as push_flux 
events are processed and the update event must be rescheduled. We know that the most recent actor state will have access to all relevant metadata, and thus we only execute the update consistent with the current submesh.t_next. All other updates become noops, either because their scheduled execution time does not match submesh.t_next or the update has already been executed.

The two functions n_outstanding_msgs and inhibits_progress examine the local representation of the distributed state of the system in order to determine what events should be scheduled next. The n_outstanding_msgs function provides a lower bound on the number of outstanding messages to be processed on the actor by time $t$. By knowing that a message will be processed before the next update time, we defer scheduling updates until the waited upon messages are received, eliminating no-ops and reducing misspeculation. The inhibits_progress function checks to see whether the actor is unable to make progress without forcing its neighbor to update. Forcing a neighbor to update may arise due to a local ordering violation, an inability to propose a CFL-stable next timestamp, or due to performance benefits of having the neighbor prematurely update.

\subsection{The Devastator Runtime}

In order to measure the performance of the ALTS algorithm, we implemented it using the Devastator PDES runtime (first described and used in [10]). Devastator is a general-purpose, optimistic PDES engine that coordinates event execution and rollback required by Jefferson's Time Warp protocol. Devastator is built using the GASNetEX runtime [3], and utilizes light-weight active messages (RPCs) to invoke events on remote logical processes. Since GASNet-EX active messages are tailored to the specific interconnect hardware, they are efficient at delivering small asynchronous events required of PDES. It is implemented with modern $\mathrm{C}++$ to improve programmer productivity, uses parallel threads and processes to enable distributed memory execution, and includes several optimizations to improve communication and synchronization efficiency. The modern $\mathrm{C}++$ interface allows clean specification of event classes, their serialization, and their execute and rollback functions.Since in general conservation laws are not reversible, portions of the actor state are logged during execute, and the actor state can be restored to the state prior to the execute call by calling the rollback function.

To illustrate the parallel efficiency of the runtime, Figure 2 shows weak scaling performance of Devastator running a modified version of the well-known PHOLD benchmark [16]. The main difference between this benchmark and the standard PHOLD algorithm is the selection of destination LP ranks using a parameterized normal distribution $(\sigma=16)$ centered at the sending LP's rank. This modification allows us to tune the communication pattern of the benchmark to more closely resemble applications with locality, such as the ALTS simulations studied here. In the figure, we show the per core event commit rate as the number of cores is varied (with the number of logical processes per core fixed at 1000). The different series in the figure vary the number of active events (rays) per logical process in the system (corresponding to the average event queue depth for each logical process). As can be seen in Figure 2, parallel efficiency (performance per core) decreases to approximately $55 \%$ to $75 \%$ as the number of cores increases from

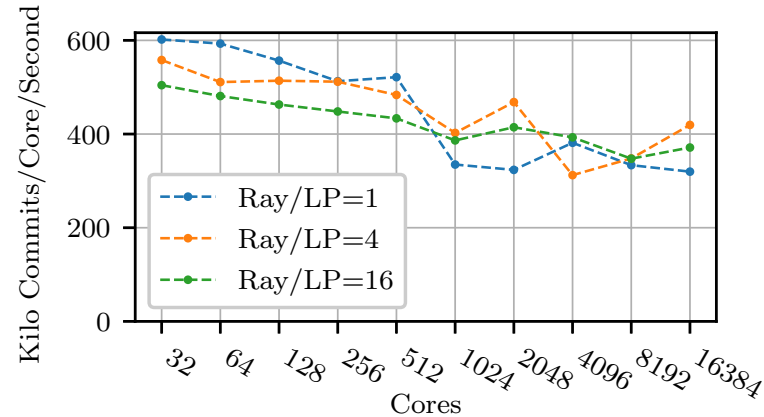

Figure 2: Weak scaling performance for the modified PHOLD benchmark on NERSC's Cori-Haswell partition.

32 cores to 16,384 cores, corresponding to a $270 \mathrm{x}$ to $380 \mathrm{x}$ overall speedup in aggregate event commit rate. The reported results are the average of three simulations. In certain cases, significant variance is observed. Nonetheless, the overall trends remain reinforced by the results at 16,384 cores, the standard deviation over average for the 3 configurations was 0.56 for 1 Ray/LP, 0.04 for 4 Rays/LP, and 0.05 for 16 Rays/LP. Determining whether the jagged nature of the scaling results is due to specific paths taken by the rays or due to system noise will be addressed in a more thorough future treatment of Devastator.

\subsection{Performance Optimizations}

Although ALTS is a mathematically stable algorithm, a naive implementation using a speculative runtime would lead to excessive overheads. In this section, we introduce a set of performance optimizations focused on eliminating bad speculation (2.2.1-2.2.4) and a dynamic load balancing scheme (2.2.5).

2.2.1 Binning timestep sizes. The need for local ordering of events leads to potentially increased forced synchronizations. For example, given submeshes stepping with timesteps 17 and 18, the submesh stepping with size 17 would step to time 17 and then have to update again at time 18 to maintain local ordering. To address this, we bin future timesteps into powers of two times a fixed $\Delta t_{\min }$. To simplify computation all timestamps are kept as integers with units of $\Delta t_{\min }$ The pythonic pseudocode for timestep binning is:

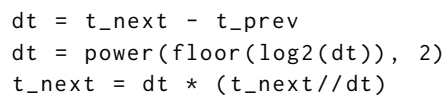

In practice, we implement this efficiently using bit shifts. The impact of this optimization is that we reduce forced updates by having submeshes with roughly the same timestep step to the same timestamps. This also concentrates updates to certain multiples of $\Delta t_{\min }$ leading to improved simulator parallelism and reduces the depth of the algorithm. The downside of this approach is that we are unable to take the largest feasible timestep potentially increasing the total number of updates.

2.2.2 Waiting on forced updates. When a submesh sends a push flux with the requires_update argument as true, we know that 
the submesh will receive a push flux from the neighboring submesh at the current time. Since any updates speculatively executed before the neighbor's push flux event has arrived would be rolled back, the function n_outstanding_msgs examines the submesh's state and delays scheduling further updates until after the waited upon messages have arrived.

2.2.3 Forcing neighbors to update. Since the CFL condition is taken relative to the last update time of the submesh or its neighbor, once a submesh updates it may still make incremental progress, enumerating the bits of the unbinned t_next. As an example, consider two submeshes both with an unbinned timestep of 14 . If one submesh is delayed, the other will execute binned updates at times 8,12 , and 14 , before being unable to make progress. Once the delayed submesh updates to time 8 , the resulting push flux will lead to rollback of 2 updates on the other submesh. To address this, we examine the difference in the proposed next timestep based on the current $t^{*}$ from the CFL condition (1) and if $t^{*}$ were equal to the submesh's current simulation time, i.e. the submeshes are synchronized. If the ratio of the synchronized timestep size versus the unsynchronized timestep size exceeds 2 , we force the submeshes causing the timestep reduction to update. In the example, the second submesh would update at time 8 , and immediately force its neighbor to update. By waiting on submeshes which have been issued push fluxes which require updating as part of Section 2.2.2, forcing the neighbor to update leads to waiting on the neighboring submesh rather than incorrectly speculatively executing updates.

2.2.4 Establishing upper bounds on neighboring updates. When $\Lambda$ and $\Delta x_{j}$ are constant but vary significantly, it should be possible to recover an efficient task-graph-like execution, where dependencies are independent of the dynamic state of the neighboring submeshes. To recover this behavior in our more general discrete-event simulation setting, we note that the next update time for a submesh must be less than the next unbinned update time required by the internal elements of that submesh. This upper bound is shared with neighboring submeshes as part of the make_metadata message. By incrementing n_outstanding_msgs if the time argument exceeds the upper bound, the neighboring submesh pauses scheduling of further events until the submesh's push flux event is processed.

2.2.5 Dynamic Load-Balancing. Lastly, as the simulation advances and simulated regions are flooding or draining, the load will need to be rebalanced. Dynamic load balancing for hurricane storm surge for synchronous timestepping has been explored in [5]. Findings indicate that while the compute load changes substantially, it does so in a slow gradual manner, and a semi-static based approach performs roughly as well as a fully asynchronous load balancing strategy. Here we use a semi-static approach due to its relative simplicity compared to the fully asynchronous load balancer.

At a high level, the simulation will be broken into epochs of a fixed size. At the end of each epoch, all load information will be aggregated on rank 0 . There the submesh to rank assignment will be recalculated to minimize load imbalance, and all submesh relocations will be issued. By fixing the element to submesh mapping and only having to assign submeshes to ranks, the complexity of the load balancing problem is considerably reduced, enabling more frequent load balancing. The load balancing problem must satisfy two constraints. Compute loads must be balanced between ranks to lead to efficient compute resource utilization, and the total number of elements assigned to a rank must not exceed available local memory. The second constraint is required since "dry" regions of the mesh do not update, so they could potentially be assigned to a single rank.

For 2D simulations such as those performed in [5], standard graph partitioning solutions such as Metis [20] are able to rebalance the problem. However, for the $1 \mathrm{D}$ problems considered in this work, we found Metis struggled to generate partitions with low load imbalance. Instead, we used a custom partitioner for this paper. We approximate the computational work of a submesh as $n_{\text {elem }}\left(1 / \Delta t-1 / \Delta t_{\text {epoch }}\right)$. Submeshes are classified as either wet or dry. A submesh is dry if it has zero work, i.e. only updates once per epoch, and is otherwise wet. Wet submeshes are partitioned by balancing the work across each rank. In addition, each rank is then assigned roughly the same number of dry submeshes (some ranks may receive an extra submesh due to discrete effects). For 1D problems, partitions can be efficiently represented with the use splitters of the global submesh graph, where rank $r$ is assigned the submeshes between the $r$-th and $(r+1)$-th splitter.

\subsection{Conservative versus Optimistic Simulation}

An alternative way to parallelize the algorithm would be through conservative PDES. Whereas Time Warp-based parallelizations have mechanisms to correct causality violations, conservative PDES requires the program to guarantee that no causality violation can occur. The most simple version of conservative PDES is to only update submeshes with a globally-determined lowest timestep. This parallelization suffers from insufficient available parallelism and significant synchronization overhead. For the hurricane storm surge problem considered in [14], a mesh with $O\left(10^{6}\right)$ elements only has $O\left(10^{4}\right)$ elements stepping in the smallest timestepping group. Amdahl's law limits the scalability of such an approach. Conservative PDES introduces more parallelism into the system through the introduction of look ahead. If for a given global timestamp, we can ensure that no causality violations occur over some time interval, all events scheduled within that interval may be safely executed. The use of a global priority queue corresponds to a look ahead of zero. To derive look ahead estimates for systems of conservation laws, we must prove that no push flux-the result of a neighbor updating-will be processed within the look ahead interval.

To achieve a viable implementation using a conservative PDES, two major problems need to be resolved.

(1) Energy-based look ahead estimates: Anticipating when submeshes update requires deriving estimates for bounding $|\Lambda|$. However, the wavespeed $|\Lambda|$ can be bounded above and below by the energy of the conservation law, a conserved derived variable. This intuition provides a useful glimpse into the evolution of $\Lambda$. Bounding the growth of $\Lambda$ would allow us to prove that no neighboring actor updates within a given time interval. However, since pathological regions of high energy could exist anywhere in the system, deriving these bounds requires non-local communication.

(2) Loosening of total variation diminishing properties: The ALTS method has been designed specifically to generate a total 
variation diminishing solution, and the numerical analysis requires submeshes to update in a locally ordered manner relative to one another. From a conservative PDES standpoint, local ordering is problematic as it may generate instantaneous event cascades, i.e. events scheduled on neighboring actors with the same timestamp offset by a small time eps. We conjecture that the need for locally ordered event traces can be eliminated by relaxing the requirement of total variation diminishing to total variation bounded solutions. Total variation bounded solutions correspond to a larger, but still stable solution class. The relaxation to total variation bounded solutions may also be inevitable as we move to higher order timestepping schemes, e.g. [11].

Given the difficulties required to obtain meaningful look ahead estimates, we have focused on optimistic simulation in this paper. Rather than focus on deriving look ahead estimates, it is simpler to derive tests which identify when speculation is guaranteed to be rolled-back based on local information and then simply wait for the message that would cause the rollback of any subsequent events to be processed.

\section{NUMERICAL EXPERIMENTS}

To describe the performance characteristics of the local timestepping algorithm, we present results for a one dimensional nonlinear shallow water equation mini-application. For this paper, we consider a first-order finite volume discretization using a local LaxFriedrichs numerical flux. To address changes in bathymetry, we use the hydrostatic reconstruction of [1]. While the real world applicability of this discretization is limited, we expect performance trends to be largely the same for 2 dimensional problems with more sophisticated discretizations. Efficient execution of ALTS fundamentally relies on amortizing Devastator overheads with useful computational work. As the number of push fluxes sent per update changes (as we go to higher dimensions, each neighbor receives one message) and local updates become more expensive due to more sophisticated discretizations, the key metrics such as minimum number of elements required per submesh may change, but the trends will ultimately remain similar.

Meshes are generated by warping uniformly distributed points on the interval $[-1,1]$. We consider a uniform mesh for which the warp function is the identity map, and a polynomial warp function, $p(x)=C\left(x^{3} / 3+\varepsilon x\right)$, where $\varepsilon=0.02$ and $C$ is chosen so that $p(1)=1$ and $p(-1)=-1$. The ratio of largest element to smallest element is approximately $\varepsilon^{-1}$. These kinds of locally refined meshes are common for fluid simulations where high accuracy is only needed near regions of interest. We specify submeshes via an iterative procedure designed to balance the work performed by each submesh during the simulation. For stencil-like kernels, the cost of performing an update is $O\left(n_{\text {elem }}\right)$. The total work per submesh is approximated as the number of elements assigned to a given submesh times the inverse binned timestep of the submesh, assuming $|\Lambda|=1$. The iterative submesh partitioning process updates the weight associated with a given element and then repartitions the submeshes. This process is repeated until a fixed point is achieved or a preset number of iterations has been computed.
We consider two problems. The first is the lake at rest (lar) problem for which the solution is a constant. This problem is notable because $|\Lambda(x, t)|$ is a constant for all space and time. Hence, any local timestepping benefit is derived solely from variation in element sizes $\Delta x_{j}$. The other problem we consider is the Carrier-Greenspan (cg) problem [8] as configured in [2]. This is a $\pi$-periodic solution to the shallow water equations which simulates water flowing up and down a sloped beach. For clarity, we have included four snapshots in Figure 3. The Carrier-Greenspan problem serves as a good proxy for hurricane storm surge. In both cases, the large variations in wavespeed $\Lambda$ are being driven by coastal flooding.

Beyond the mesh, submeshes, and initial conditions, we need to specify a few inputs. The smallest timestep size is set through the parameter $(|\Lambda| / \Delta x)_{\max }$, which specifies the largest expected value of $|\Lambda| / \Delta x_{j}$ during the simulation. Note that setting this parameter is not very onerous, as the simulation will still perform well if this value is set significantly below the true $(|\Lambda| / \Delta x)_{\max }$. This value determines the smallest timestep size, by quantizing the simulation in $2\left\lceil t_{\text {end }}(|\Lambda| / \Delta x)_{\max }\right\rceil$ steps. The extra factor of 2 is the inverse of the $C_{C F L}$ factor required for total variation diminishing timestepping. The minimum timestep size is set to $t^{\text {end }} / n_{\text {steps }}$. Additionally, we specify the epoch time as $\Delta t_{\text {epoch }}$. To avoid issues with timestep binning, $\Delta t_{\text {epoch }}$ is rounded down to the nearest power of 2 times $\Delta t_{\text {min }}$.

All numerical results here are run on NERSC's Cori-Haswell partition. Each node consists of 2 Intel Xeon Processors E5-2698 v3. Each Xeon processor has 16 cores. When running devastator, there are two parallel settings. (world=threads) runs with a single process and uses shared memory parallelization where each thread participates in executing simulation events. (world=gasnet) is used for distributed simulation with multi-threaded processes. In this case, one thread per process is dedicated to sending and receiving messages, while the remaining worker threads execute simulation events. To avoid NUMA effects, we assign a separate process to each of the two processors on a node.

\subsection{Event Overheads}

What makes ALTS different from traditional PDES applications is that we can freely manage event granularities by tuning the number of elements assigned to each submesh. We solve the lake at rest problem on a fixed mesh with 20,000 elements for the uniform mesh and 75, 000 elements for the polynomial mesh. The simulation is run varying the number of submeshes, ranging from 1 to 8 submeshes per worker thread. As the number of submeshes increases, the concurrency available to the discrete event simulator increases (i.e. over-decomposition), allowing useful work to occur while waiting for messages to arrive. Furthermore, each submesh must satisfy the CFL condition for fewer elements, leading to a more optimal local CFL condition. This is an important performance tuning knob for irregular algorithms, as we are able to weigh the benefits of increased irregularity versus reduced PDES overheads.

We ran the following experiments on 64 cores on Cori. Each run consists of 4 processes-one per NUMA domain-with 15 worker threads per process. Enumerating the submeshes from left to right, we assign submeshes in contiguous chunks, i.e. rank $k$ is assigned submeshes $C_{F} k$ through $C_{F}(k+1)$, where $C_{F}$ is the number of 


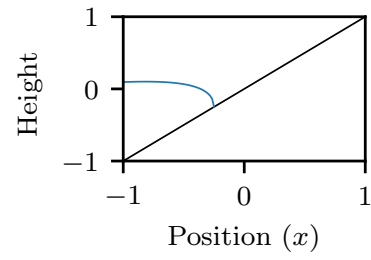

(a) $t=0$

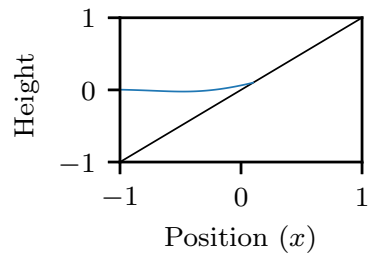

(b) $t=\pi / 4$

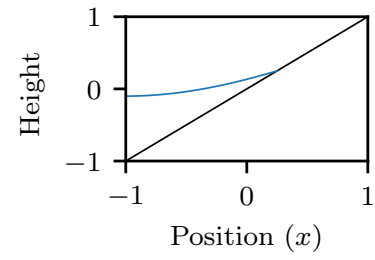

(c) $t=\pi / 2$

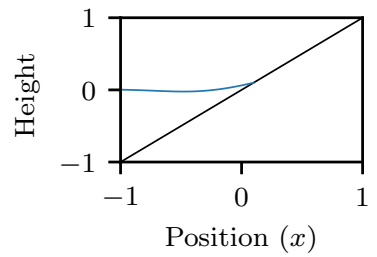

(d) $t=3 \pi / 4$

Figure 3: Water surface height (in blue) and seafloor (in black) relative to mean geoid for the Carrier-Greenspan problem at various time points.

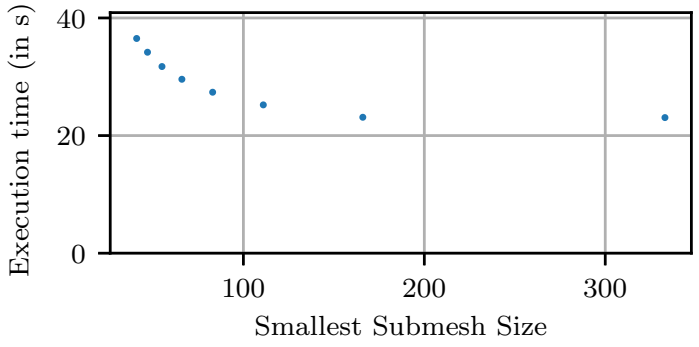

(a) Uniform

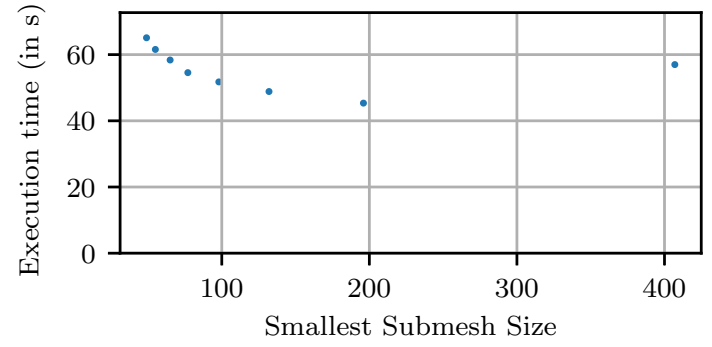

(b) Polynomial

Figure 4: Execution time versus smallest number of elements assigned to a submesh.

submeshes per rank. The critical path will lie on the rank with the submeshes with the fewest elements. Since the work performed per submesh is balanced for the lake at rest problem, the ALTSrelated work completed on this rank is comparable to that of other ranks. However, this rank must execute more updates and therefore, incurs the more event overheads.

In Figure 4, we plot execution time versus the lowest number of elements assigned to a submesh. We selected the simulation time to be sufficiently long so that reliable measurements could be made. We set $t^{\text {end }}=64,(|\Lambda| / \Delta x)_{\max }=20200$ for the uniform mesh, and $t^{\text {end }}=1,(|\Lambda| / \Delta x)_{\max }=1115208$ for the polynomial mesh. Each configuration was run 5 times and the standard deviation of each set of runs was within $0.99 \%$ of the respective means. For either configuration, we see that once the element size per submesh drops below 70 elements, event overheads begin to dominate the execution time. While there is a slight benefit to oversubscribing compute resources for the uniform mesh, for the polynomial mesh we observe a speedup of 1.26 going from an oversubscription factor of 1 (407 minimum elements per submesh) to 2 (196 minimum elements per submesh). For the uniform mesh, dependencies are approximately satisfied at the same time, but for the polynomial mesh, a neighboring submesh which is substepping must first make progress before the submesh with the larger timestep is able to progress causing the performance degradation for the largest minimum submesh in Figure 4b. Oversubscription allows us to update a different submesh while waiting for the substepped submesh to satisfy the stalled submesh's dependencies.
Lastly, we build a cost model which approximates the time elapsed updating a submesh of $n_{\text {elem }}$. Since the cost of the update kernel is $O\left(n_{\text {elem }}\right)$, we assume a linear model of the form $\operatorname{cost}\left(n_{\text {elem }}\right)=C_{2} n_{\text {elem }}+C_{1}$, where $C_{2}$ to correspond to the cost to update one element and $C_{1}$ to roughly be the event overhead. Using the data for the polynomial mesh runs and omitting the data point for an oversubscription factor of one due to the aforementioned discrete affects impeding performance, we estimate the average task execution time by dividing the overall execution time by the largest number of updates committed on any rank, and then use the average number of elements per submesh on that rank as the $n_{\text {elem }}$ input. Due to the smooth nature of the mesh refinement, we assume all submeshes on the selected rank update the same number of times. Via linear regression, we find $C_{2}=0.0438 \mu \mathrm{s} / \mathrm{element}$ and $C_{1}=1.474 \mu \mathrm{s}$ with $R^{2}=0.999$. From this cost model, we approximate that roughly 100 elements are needed to achieve a good resource utilization. At this task granularity, $74.8 \%$ of the time is spent doing useful work. This corresponds to an event size of $5.9 \mu \mathrm{s}$.

\subsection{Description of Misspeculation}

To explore when and where speculation is occurring we consider a single node (world=threads) problem with a 300,000 element mesh for both uniform and polynomial meshes as well as the lake at rest and Carrier-Greenspan problems. Submeshes are oversubscribed by a $6 \mathrm{X}$ oversubscription factor. We use a trivial actor partitioning, leading to imbalanced work-to-rank assignments. However, as a study of speculation, this serves as a stress test since underworked 
ranks have the possibility to do more work that will eventually be rolled back while waiting on messages from overworked neighbors.

For the lake at rest problem, we observed no rollback of updates during the simulation. For this problem, the only source of variation in timestep sizes is due to variation in element sizes, not the local wavespeed. Insofar, the information sent via the upper bound messages anticipates when messages will arrive and allows the submesh to avoid performing updates which would be rolled back.

For the Carrier-Greenspan problem, the amount of speculation remains small. Only $0.005 \%$ of element updates for the uniform mesh and $0.006 \%$ of element updates for the polynomial mesh were reversed. Furthermore, in Figure 5, we compare on which ranks rollback occurred as a function of wall clock time versus the expected timestep size based on the analytical solution of the Carrier-Greenspan problem. The occurrence of rollback occurs at the interfaces of submeshes stepping with different step sizes.

The occurrence of speculation is fairly limited in all of these configurations. When the local prediction of incoming messages is accurate, we are able to prevent bad speculation by waiting on the incoming message rather than executing updates which will be inevitably rolled back, the execution of the local timestepping algorithm is equivalent to that of the task-graph based implementations of local timestepping for linear problems as described in Section 2.2.4. Furthermore, these experiments showcase the latent irregularity in the application. We see that timestep changes are relatively infrequent and localized, although they may move throughout the domain. The benefits of speculation are less about doing useful work while being uncertain if it will need to be rolled back, but rather avoiding paying the expensive synchronization overheads required to prove that no causality violations occur.

\subsection{Strong Scaling Comparison to STS-MPI}

We turn to distributed performance with a strong scaling study of the Carrier-Greenspan problem on a polynomial mesh with 1.2 million elements. We compare the performance of the ALTS Devastator (ALTS-deva) to a synchronously stepped MPI implementation (STS-MPI). At 1.2 million elements, the smallest submesh size is 99 elements, limiting devastator overheads per Section 3.1. For the MPI version, each submesh steps at each timestep i.e. synchronously. However, the STS-MPI implementation utilizes point-to-point nonblocking messages and overlaps communication with available local work. Furthermore, both implementations use the same actor data structures and internal helper functions to ensure a fair comparison.

Since ALTS improves performance via work reduction, we use a throughput measure which approximates the throughput as work done for a synchronous problem divided by the execution time, i.e.

$$
T P=\frac{t^{\mathrm{end}} n_{\mathrm{elem}}}{\Delta t^{\mathrm{min}} T_{X}^{n}},
$$

where $t^{\text {end }}=2 \pi$ is the simulation time, $n_{\text {elem }}=1.2 \cdot 10^{6}$ is the number of elements in the mesh, $\Delta t_{\min }=7.8125 \cdot 10^{-8}\left((|\Lambda| / \Delta x)_{\max }=\right.$ $\left.6.4 \cdot 10^{6}\right)$ is the smallest timestep taken, and $T_{X}^{n}$ is the execution time measured in seconds with $n$ cores using parallelization $X-$ either Devastator or MPI. Figure 6 compares the output of the MPI-synchronous timestepping (STS-MPI) implementation to the
Devastator-ALTS (ALTS-deva) implementation. For the MPI implementation, the 32 core run has been omitted since it was unable to finish within queue time constraints. The core counts are chosen to highlight the scaling behavior of the ALTS-deva implementation. At 1024 cores, the oversubscription factor is 3 submeshes per rank. Scaling out further without modifying submesh sizes would lead to discrete load balancing issues, degrading performance. Since ALTS achieves its speed-up through work reduction, we observe an improvement in per-core throughput when run on 128 to 1024 cores, rather than an improvement in the maximum number of cores to which we are able to scale out. At 128 cores, the ALTS-deva implementation has a speed-up of 3.75x over the STS-MPI implementation. This speed-up degrades to $2.57 \mathrm{x}$ at 768 cores. Nonetheless, at 768 cores, the ALTS-deva implementation achieves a time to solution comparable to the 2,048 core STS-MPI configuration.

This strong scaling study is designed to show tangible benefits of ALTS to accelerate effective per core throughput. However, the more regular workload of the STS-MPI application remains more scalable. We have shown that the 2,048 core MPI run matches the best throughput of the ALTS-deva implementation. At 6,144 cores, the STS-MPI implementation has a parallel efficiency of $80 \%$ and a speed-up over the best ALTS-deva time to solution of 2.42. This discrepancy is important to emphasize. If time-to-solution remains the primary objective, synchronous timestepping remains the best option. However, when using limited compute resources, ALTS can significantly outperform synchronous timestepping provided that the problem is large enough.

The speed-ups obtained are ultimately determined by the distribution of element sizes $\left\{\Delta x_{j}\right\}$ and problem dynamics. Taking the ratio of element updates for the synchronous timestepping method divided by the number of element updates for adaptive local timestepping, we find a theoretical speed-up based on work estimates of $S^{\text {work }}=5.38$. The remainder of this section will focus on explaining the discrepancy between the observed speed-ups between the STS-MPI implementation and the ALTS-deva implementation. There are 5 contributors worth examining when comparing the two implementations:

(1) Time spent load balancing: Due to Amdahl's law as well as the growing cost of the load balancing problem as the number of ranks increases, increasing amounts of time may be spent inside of the load balancing phase rather than advancing the state of the system. Between the 32 core and 512 core configuration, time spent load balancing went from 15 seconds to 17.9 seconds, a modest increase but less than $1 \%$ of the total execution time.

(2) ALTS-overhead: The cost of computing the CFL condition for the shallow water equations requires evaluation of a square root, which creates considerable algorithmic overhead. These computations are omitted for the STS-MPI implementation, which steps with a fixed step size. Since $|\Lambda|$ is constant for the lake at rest problem, we run the problem with and without the CFL computation on one node. To avoid running into issues with Devastator overhead, we partition the 1.2 million element mesh into 180 actors. Comparing the execution times, we determine $25 \%$ of the execution time is spent on the CFL computation. 


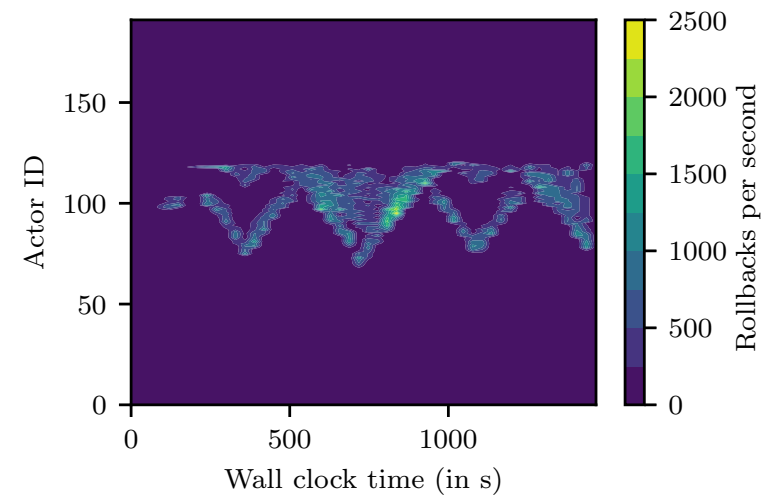

(a) Rollback versus wall clock time

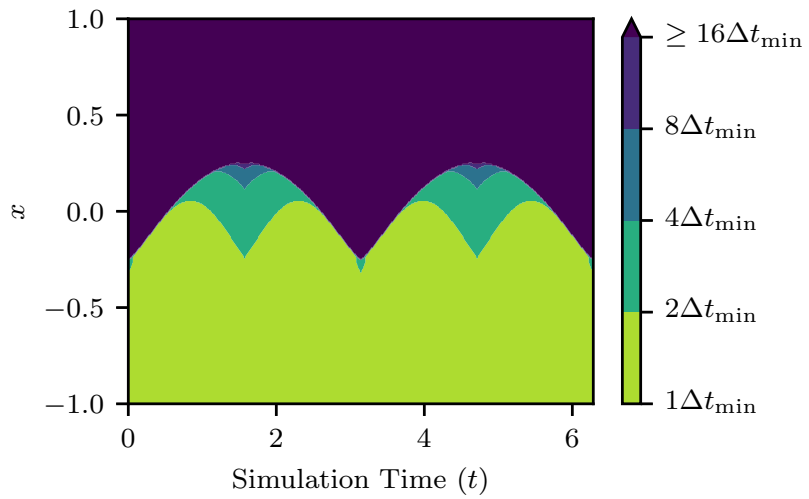

(b) Theoretical timestep size

Figure 5: Rollback and timestep size for the Carrier-Greenspan problem on the uniform mesh.

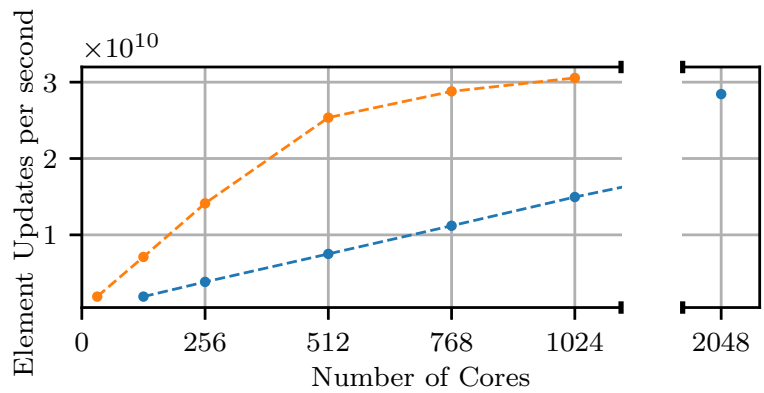

Figure 6: Comparison of throughput $(T P)$ versus number of cores for the ALTS-deva implementation o versus the STSMPI implementation o.

(3) Rollback: When bad speculation is detected, the updates performed will have to be undone, and the time spent executing those updates is essentially wasted. Similar to Section 3.2, only very few updates are rolled back. At 32 cores, $0.005 \%$ of element updates were rolled back, and at 512 cores, $0.009 \%$ of element updates were rolled back, suggesting that our performance optimization heuristics do a good job of identifying and preventing mis-speculation.

(4) Event-scheduling overhead: The minimum mesh size has been chosen to limit the impact of Devastator runtime event scheduling overheads. These overheads are small but non-trivial and may degrade performance if many small submeshes are on the critical path.

(5) Imbalance: Given the fact that the load balancer must assign submeshes (rather than elements) to ranks as well as the dynamic changes in the work associated with each submesh, the load balance will not be perfect after each load balancing phase. Using the imbalance of the problem going into and out of the load balancing phase we can approximate the time averaged imbalance during the simulation, i.e.
$I=\left(w_{a^{*}}-\overline{w_{a}}\right) / \overline{w_{a}}$ where $w_{a^{*}}$ is the maximum work per rank and $\overline{w_{a}}$ is the average work. At 32 cores, the problem is well balanced with $I=0.02$. The load imbalance for the 512 core run worsens to 0.43 . This is partially to be expected as at 512 cores, there are only 6 submeshes per rank. With only half the mesh being wet on average, the number of submeshes available for load balancing is effectively halved. However, this imbalance is more pessimistic than the observed performance, i.e. a parallel efficiency of $83.7 \%$. This discrepancy arises due to a mismatch between the actual work associated with a submesh and our cost model outlined in Section 2.2.5. Firstly, Devastator overheads, i.e. performance costs which are fixed per submesh are ignored in the load balancing problem. Secondly, as we strong scale, cache effects may be causing the code to run faster than expected. Nonetheless, the imbalance metric indicates that the load balancer begins to struggle as we strong scale to fewer submeshes per thread. Although there exist discrepancies between the modeled imbalance and the actual imbalance, the fact that the 32 core configuration has low imbalance and performs well suggests that our approach is able to effectively balance compute loads across ranks.

(6) Worker thread mismatch: Another loss of performance is caused by the fact that Devastator dedicates one thread to managing communication per socket. Thus, per node on Cori, ALTS-deva only uses 30 worker threads to execute events, whereas STS-MPI uses all 32 cores.

Based on these analyses, we determine that the primary drivers of performance loss are computing the CFL condition and worsening load balance. Nonetheless, at low core counts, the problem can be sufficiently balanced, and the ALTS-deva implementation achieves throughput improvements of up to $4 \mathrm{x}$ over the STS-MPI implementation, which is $74.5 \%$ of the peak theoretical speed-up.

\subsection{Weak Scaling Performance}

To show that ALTS can be scaled to larger distributed problems, we perform a weak scaling study using the Carrier-Greenspan problem 


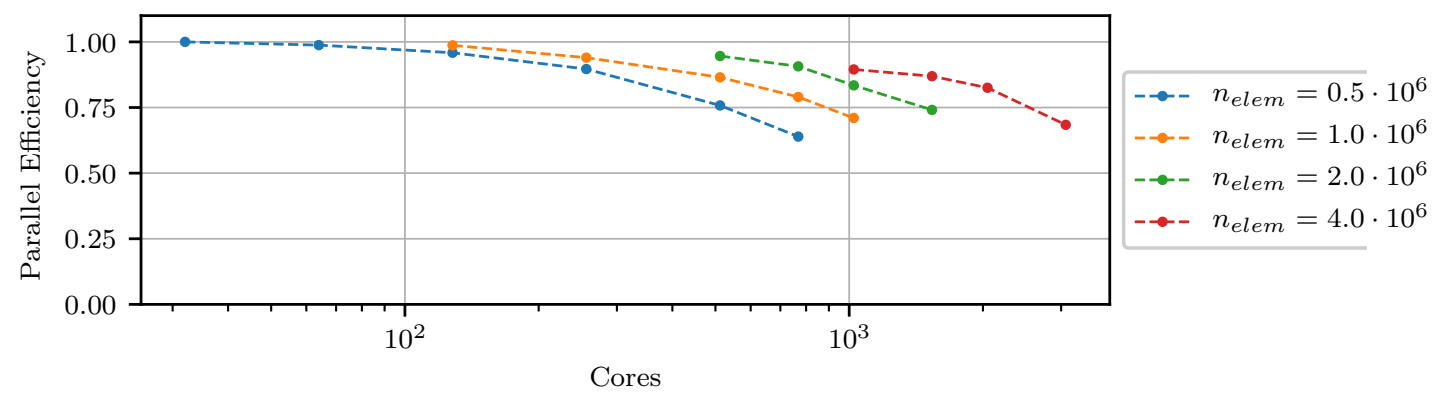

Figure 7: Weak scaling performance for the Carrier-Greenspan problem on the uniform mesh.

on the uniform mesh. Since the performance of the ALTS method strongly depends on the distribution of the wavespeed, $|\Lambda|$, it is important to simulate the full $2 \pi$ time to compare performance across different problem sizes. Due to the fact that the amount of computational work scales quadratically with the number of elements, and the fact that the polynomial mesh is computationally too expensive, using the uniform mesh allows us to consider a larger range of problem sizes.

We consider 4 submeshes with 500 thousand, 1 million, 2 million, and 4 million elements. The problem is partitioned into 2880 actors per 500,000 elements. This leads to a minimum submesh size of 174 elements, ensuring that simulator overheads are reasonably amortized. The rebalance frequency is taken to be once every $8192 \Delta t_{\text {min }}$ As the number of elements doubles, $\Delta t_{\min }$ is halved, and the total number of load balancing phases doubles. With the work per core per epoch remaining constant across weak scaling configurations, we observed that this rebalance frequency provided the optimal performance.

Each configuration is run over a few core counts to demonstrate strong scaling characteristics. The parallel efficiency versus core count is shown in Figure 7. The simulation weak scales well with the 4 million element mesh obtaining $90 \%$ of ideal per-core throughput at 1,024 cores and $68 \%$ of ideal per-core throughput at 3,072 cores. Looking at the imbalance across the runs, we see that the average imbalance for the 32 core 500 thousand element mesh was 0.03 . For the 4 million element mesh, the imbalance increases to 0.14 for the 1,024 core configuration to 0.33 for the 3,072 core configuration, leading to a slight degradation in scalability. The choice of a uniform mesh will lead to higher fractions of elements updating each timestep than a polynomial mesh. Thus, the average number of elements per core may not be a good indicator of work required to achieve good scaling behavior, nonetheless we conclude that if the problem is load balanced and sufficiently large, the ALTS method can be scaled out.

\subsection{Impact of Performance Optimizations}

To help quantify the impact of the performance optimization strategies described in Section 2.2, we consider a few problems and then enable the strategies to observe their impact. This section is split into two sections highlighting the benefits of waiting on misspeculation and load balancing.

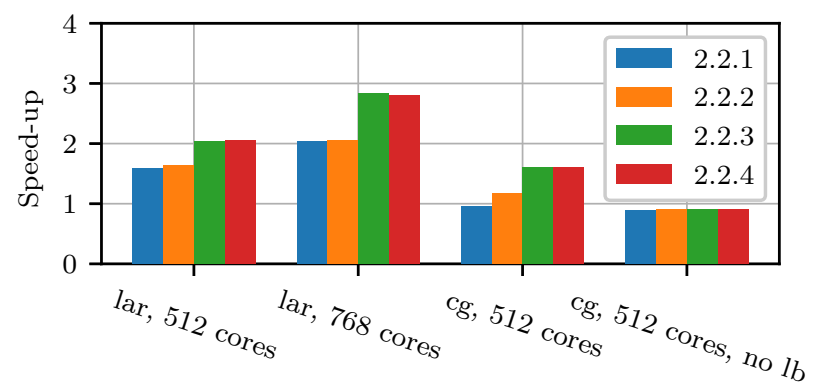

Figure 8: Speed-up obtained by incrementally enabling optimizations identified by subsection number.

3.5.1 Waiting Heuristics. To determine the impact of the performance optimizations outlined in Sections 2.2.1-2.2.4, we incrementally enable them and report the impact on speed-ups relative to a local timestepping baseline with no waiting heuristics enabled. We consider a 512 core lake at rest problem $\left(n_{\text {elem }}=\right.$ $\left.6 \cdot 10^{5},(|\Lambda| / \Delta x)_{\max }=1370368000, t^{\text {end }}=0.1, n_{\text {actors }}=1440\right)$ and 768 core lake at rest problem $\left(n_{\text {elem }}=1.2 \cdot 10^{6},(|\Lambda| / \Delta x)_{\max }=\right.$ $\left.2740736000, t^{\text {end }}=0.05, n_{\text {actors }}=1440\right)$ as well as the 512 core configuration used in the strong scaling study. To avoid exaggerating the impact of the waiting heuristics, these problems have been sized to sustain a reasonable parallel efficiency. The lake at rest configurations achieve $90 \%$ of the throughput rate of lake at rest problems with twice the number of elements. We also consider two Carrier-Greenspan configurations, one which exactly mimics the strong scaling configuration, and another without load balancing. The cumulative speed-ups for each configuration relative to a baseline without any optimizations enabled are presented in Figure 8. For these bar graphs, cumulative implies that the preceding optimizations remain enabled, e.g. the speed-up for Section 2.2.3 has also enabled Section 2.2.1 and Section 2.2.2. For the lake at rest problems, we observe the largest benefits occur when binning timesteps to powers of 2 (Section 2.2.1) and forcing neighbors to update (Section 2.2.3). The $(|\Lambda| / \Delta x)_{\max }$ parameter for these configurations has been chosen to be intentionally small, leading to significant lengthening of the critical path as outlined in Section 2.2.1. For the Carrier-Greenspan configurations, $(|\Lambda| / \Delta x)_{\max }$ is chosen to be relatively close to the optimal value. This reduces the extra updates 


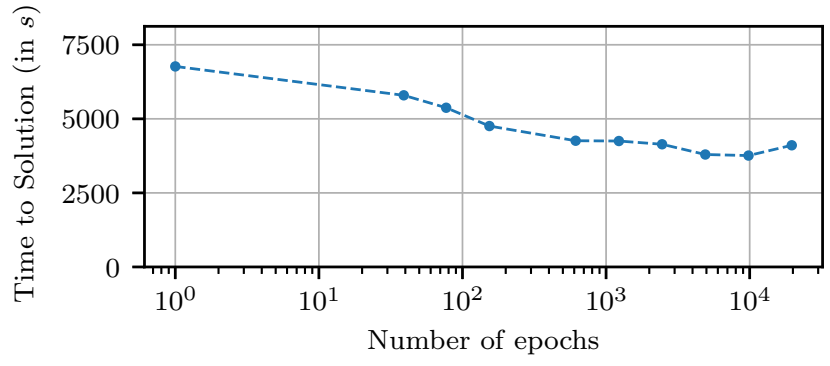

Figure 9: Impact of load balancing frequency on time to solution.

associated with enforcing local ordering since without Section 2.2.1, timesteps are binned into multiples of $\Delta t_{\min }$ (rather than $2^{k} \Delta t_{\min }$ ). In conjunction with a more optimal timestep taken at the critical path, we observe that the time to solution slightly increases after binning timesteps into powers of 2 .

Another take away from these results is that misspeculation impacts time to solution only when it delays the critical path. Since rollback is triggered by arrival of delayed push fluxes from neighboring submeshes, when the simulation is very imbalanced, the rank which is on the critical path has the slowest rate of advancing the simulation time, and as this rank makes progress, messages that would incur rollback have most likely already arrived. The unbalanced Carrier-Greenspan configuration with no performance optimizations enabled executed 1.3x more element updates than the configuration with all optimizations enabled, yet ran $10 \%$ faster. Only when considering load balanced problems, either through construction (lake at rest) or via load balancing (Carrier-Greenspan) do we observe performance improvements by reducing misspeculation. In these cases, the rollback-causing messages have not necessarily arrived and anticipating and waiting on these messages when feasible, leads to improved time to solution. For the 768 core lake at rest problem, the percentage of the cell updates rolled back on the critical path goes down from $46 \%$ down for the Section 2.2.1 configuration to zero for the fully optimized case.

3.5.2 Load Balancing. For the 512 core strong scaling problem, load balancing plays a significant role, delivering a speed-up of $1.8 \mathrm{x}$ over a run without dynamic load balancing. In Figure 9, we compare the impact of rebalancing frequency on time to solution. We note that the problem has a relatively wide minimum. With only 614 epochs, the time to solution is only $13 \%$ larger than the optimal time to solution, which occurs with 9,818 epochs. To understand this behavior, note that the worst case imbalance corresponds to when all submeshes on a rank are wet. In that sense, once the rebalance frequency begins to significantly exceed the rate of flooding, the problem becomes effectively balanced.

Thus, it is important to emphasize that although the algorithm is and must be stable under very rapidly changing values of $|\Lambda|$, these scenarios are rarely seen in practice. Rather we see gradual shifts in timestepping group membership, that if not accounted for will lead to instabilities, but are nevertheless gradual enough that these simulations can be load balanced effectively. Although, we have only considered a mini-app in this paper, we note that to a large extent these results comprise the worst case scenarios. Rarely, would all inundation be concentrated in a single submesh, rather inundation would be spread out over a large front, lowering the overall rate of imbalance generation.

\section{CONCLUSION}

In this paper, we analyze the parallel performance of adaptive local timestepping using a Timewarp-based optimistic parallel discrete event simulation. We introduce several performance optimizations, including load balancing and waiting heuristics, for the ALTS implementation that together provide a $1.6 \mathrm{x}$ to $2.8 \mathrm{x}$ speedup over a baseline PDES implementation. With these optimizations, we enable ALTS to achieve up to $4 \mathrm{x}$ speed-up $(74.5 \%$ of the theoretical speed-up) in time to solution over the state-of-the-art MPI parallelization and demonstrate weak scaling over core counts of interest to hurricane storm surge forecasting. Furthermore, analysis of misspeculation shows that for fluid simulations with gradually locally refined meshes and sufficiently large event sizes, rollback has a minor impact on performance. This finding should extend to a large class of shallow water simulations and highlights the fact that in practice changing of timesteps is limited and gradual. With this limited irregularity and heuristics to identify the majority of misspeculation, we conclude that optimistic parallel discrete event simulation provides an efficient parallelization of ALTS. Future work includes extending the algorithm to higher order discretizations and examining the performance of the method for real-time forecasting scenarios.

\section{ACKNOWLEDGMENTS}

This manuscript has been authored by an author at Lawrence Berkeley National Laboratory under Contract No. DE-AC02-05CH11231 with the U.S. Department of Energy. The U.S. Government retains, and the publisher, by accepting the article for publication, acknowledges, that the U.S. Government retains a non-exclusive, paid-up, irrevocable, world-wide license to publish or reproduce the published form of this manuscript, or allow others to do so, for U.S. Government purposes.

Additionally, this work was supported by the National Science Foundation under Grant NSF 1854986.

\section{REFERENCES}

[1] Emmanuel Audusse, François Bouchut, Marie-Odile Bristeau, Rupert Klein, and Benoît Perthame. 2004. A fast and stable well-balanced scheme with hydrostatic reconstruction for shallow water flows. SIAM Journal on Scientific Computing 25, 6 (2004), 2050-2065.

[2] Onno Bokhove. 2005. Flooding and Drying in Discontinuous Galerkin FiniteElement Discretizations of Shallow-Water Equations. Part 1: One Dimension. Journal of Scientific Computing 22, 1 (2005), 47-82.

[3] Dan Bonachea and Paul H. Hargrove. 2018. GASNet-EX: A High-Performance, Portable Communication Library for Exascale. In Proceedings of Languages and Compilers for Parallel Computing (LCPC'18) (Lecture Notes in Computer Science, Vol. 11882). Springer International Publishing, 138-158. https://doi.org/10.25344/ S4QP4W Lawrence Berkeley National Laboratory Technical Report (LBNL2001174).

[4] Maximilian Bremer. 2020. Task-Based Parallelism for Hurricane Sotrm Surge Modeling. Ph.D. Dissertation. The University of Texas at Austin.

[5] Maximilian H. Bremer, John D. Bachan, and Cy P. Chan. 2018. Semi-Static and Dynamic Load Balancing for Asynchronous Hurricane Storm Surge Simulations. In Proceedings of the Parallel Applications Workshop, Alternatives To MPI (PAWATM '18). IEEE Computer Society, Los Alamitos, CA, USA, 44-56. 
[6] Alexander Breuer, Alexander Heinecke, and Michael Bader. 2016. Petascale Local Time Stepping for the ADER-DG Finite Element Method. In International Paralle and Distributed Processing Symposium (IPDPS '16). IEEE Computer Society, Los Alamitos, CA, USA, 854-863.

[7] Steven R. Brus, Damrongsak Wirasaet, Ethan J. Kubatko, Joannes J. Westerink, and Clint Dawson. 2019. High-order discontinuous Galerkin methods for coastal hydrodynamics applications. Computer Methods in Applied Mechanics and Engineering 355 (2019), 860 - 899.

[8] George F. Carrier and Harvey P. Greenspan. 1958. Water waves of finite amplitude on a sloping beach. Journal of Fluid Mechanics 4, 1 (1958), 97-109.

[9] François E Cellier, Ernesto Kofman, Gustavo Migoni, and Mario Bortolotto. 2008. Quantized state system simulation. Proc. GCMS'08, Grand Challenges in Modeling and Simulation (2008), 504-510.

[10] Cy Chan, Bin Wang, John Bachan, and Jane Macfarlane. 2018. Mobiliti: scalable transportation simulation using high-performance parallel computing. In 2018 21st International Conference on Intelligent Transportation Systems (Maui, HI) (ITSC). IEEE, New York, NY, USA, 634-641.

[11] Emil M. Constantinescu and Adrian Sandu. 2007. Multirate Timestepping Methods for Hyperbolic Conservation Laws. Fournal of Scientific Computing 33, 3 (01 December 2007), 239-278.

[12] Clint Dawson and Robert Kirby. 2001. High Resolution Schemes for Conservation Laws with Locally Varying Time Steps. SIAM Journal on Scientific Computing 22, 6 (2001), 2256-2281.

[13] Clint Dawson, Ethan J. Kubatko, Joannes J. Westerink, Corey Trahan, Christopher Mirabito, Craig Michoski, and Nishant Panda. 2011. Discontinuous Galerkin methods for modeling hurricane storm surge. Advances in Water Resources 34, 9 (2011), 1165-1176.

[14] Clint Dawson, Corey Jason Trahan, Ethan J. Kubatko, and Joannes J. Westerink. 2013. A parallel local timestepping Runge-Kutta discontinuous Galerkin method with applications to coastal ocean modeling. Computer Methods in Applied Mechanics and Engineering 259 (2013), 154 - 165.

[15] Xenofon Floros, Federico Bergero, François E Cellier, and Ernesto Kofman. 2011. Automated simulation of modelica models with QSS methods-the discontinuous case. In 8th International Modelica Conference. 657-667.

[16] Richard M Fujimoto. 1990. Parallel discrete event simulation. Commun. ACM 33 10 (1990), 30-53.

[17] Ernst Hairer, Syvert P. Nørsett, and Gerhard Wanner. 1993. Solving Ordinary Differential Equations I. Springer-Verlag, Berlin Heidelberg.

[18] Thi-Thao-Phuong Hoang, Wei Leng, Lili Ju, Zhu Wang, and Konstantin Pieper 2019. Conservative explicit local time-stepping schemes for the shallow water equations. f. Comput. Phys. 382 (2019), $152-176$.
[19] Jen-Chih Huang, Xiangmin Jiao, Richard M. Fujimoto, and Hongyuan Zha. 2007. DAG-guided Parallel Asynchronous Variational Integrators with Super-elements. In Proceedings of the 2007 Summer Computer Simulation Conference (San Diego, California) (SCSC '07). Society for Computer Simulation International, San Diego, CA, USA, 691-697.

[20] George Karypis and Vipin Kumar. 1998. A fast and high quality multilevel scheme for partitioning irregular graphs. SIAM fournal on Scientific Computing 20, 1 (1998), 359-392.

[21] Ernesto Kofman and Sergio Junco. 2001. Quantized-state systems: a DEVS Approach for continuous system simulation. Transactions of The Society for Modeling and Simulation International 18, 3 (2001), 123-132.

[22] Lilia Krivodonova. 2010. An efficient local time-stepping scheme for solution of nonlinear conservation laws. f. Comput. Phys. 229, 22 (2010), $8537-8551$.

[23] Randall J. LeVeque. 1992. Numerical methods for conservation laws. Birkäuser Verlag, Basel.

[24] A. Lew, J. E. Marsden, M. Ortiz, and M. West. 2003. Asynchronous Variational Integrators. Archive for Rational Mechanics and Analysis 167, 2 (01 April 2003), 85-146.

[25] Yuri A. Omelchenko and Homa Karimabadi. 2006. Event-driven, hybrid particlein-cell simulation: A new paradigm for multi-scale plasma modeling. f. Comput. Phys. 216, 1 (2006), 153 - 178.

[26] Yuri A. Omelchenko and Homa Karimabadi. 2007. A time-accurate explicit multi-scale technique for gas dynamics. 7. Comput. Phys. 226, 1 (2007), 282-300.

[27] Yuri A. Omelchenko and Homa Karimabadi. 2012. HYPERS: A unidimensional asynchronous framework for multiscale hybrid simulations. 7. Comput. Phys. 231, 4 (2012), 1766 - 1780

[28] Stanley Osher and Richard Sanders. 1983. Numerical Approximations to Nonlinear Conservation Laws with Locally Varying Time and Space Grids. Math. Comp. 41, 164 (1983), 321-336.

[29] John R Rice. 1960. Split Runge-Kutta Method for Simultaneous Equations. Fournal of Research of the National Bureau of Standards: Mathematics and mathematical physics. B 64B, 3 (1960), 151-170.

[30] Martin Schlegel, Oswald Knoth, Martin Arnold, and Ralf Wolke. 2009. Multirate Runge-Kutta schemes for advection equations. 7. Comput. Appl. Math. 226, 2 (2009), 345 - 357. Special Issue: Large scale scientific computations

[31] Carsten Uphoff, Sebastian Rettenberger, Michael Bader, Elizabeth H. Madden, Thomas Ulrich, Stephanie Wollherr, and Alice-Agnes Gabriel. 2017. Extreme Scale Multi-physics Simulations of the Tsunamigenic 2004 Sumatra Megathrust Earthquake. In Proceedings of the International Conference for High Performance Computing, Networking, Storage and Analysis (Denver, Colorado) (SC '17). ACM, New York, NY, USA, Article 21, 16 pages. 